\title{
Sensitive detection of nanomechanical motion using piezoresistive signal downmixing
}

\author{
I. Bargatin, E. B. Myers, J. Arlett, B. Gudlewski, and M. L. Roukes \\ Condensed Matter Physics 114-36, California Institute of Technology, Pasadena, California 91125
}

(Received 27 September 2004; accepted 14 February 2005; published online 24 March 2005)

\begin{abstract}
We have developed a method of measuring rf-range resonance properties of nanoelectromechanical systems (NEMS) with integrated piezoresistive strain detectors serving as signal downmixers. The technique takes advantage of the high strain sensitivity of semiconductor-based piezoresistors, while overcoming the problem of rf signal attenuation due to a high source impedance. Our technique also greatly reduces the effect of the cross-talk between the detector and actuator circuits. We achieve thermomechanical noise detection of cantilever resonance modes up to $71 \mathrm{MHz}$ at room temperature, demonstrating that downmixed piezoresistive signal detection is a viable high-sensitivity method of displacement detection in high-frequency NEMS. () 2005 American Institute of Physics. [DOI: 10.1063/1.1896103]
\end{abstract}

The field of microelectromechanical systems (MEMS) has received increased attention in recent years in both the scientific and technological realms. Recent experimental efforts have expanded this field into the nanometer-scale regime [so-called nanoelectromechanical devices, or NEMS ${ }^{1}$ ], increasing operating frequencies to the megahertz ${ }^{2,3}$ or even gigahertz ${ }^{4}$ range. At such frequencies a host of new applications become possible, from sensitive charge detection ${ }^{5}$ to mass sensing, ${ }^{6}$ biological imaging, ${ }^{7}$ and quantum measurement. $^{8}$

Current methods for displacement detection in NEMS include magnetomotive, ${ }^{2}$ optical interferometric, ${ }^{3}$ and single electron transistor ${ }^{8}$ techniques. An alternate method that has proved useful for microscale, but not yet nanoscale, devices utilizes piezoresistive strain sensors integrated directly into the device. Piezoresistors have previously been incorporated into microscale cantilevers ${ }^{9,10}$ and used, for example, in atomic force microscopy, ${ }^{9}$ data storage, ${ }^{11}$ and biosensing. ${ }^{12}$

These devices achieve great strain sensitivity by using semiconductor-based piezoresistors, primarily doped $\mathrm{Si}^{13}$ or AlGaAs. ${ }^{14}$ Compared with other detection methods, piezoresistive NEMS have the advantages of being fully integrated sensors that operate from room temperature down to at least $4 \mathrm{~K}$ and do not require magnetic field. Resonance detection of cantilevers up to $9 \mathrm{MHz}$ has been achieved previously using doped Si piezoresistors. ${ }^{11}$ However, it was proved difficult to extend this technique further to NEMS due to the intrinsically high resistances $(5-100 \mathrm{k} \Omega$ ) of these devices, which leads to frequency-dependent signal attenuation at $\mathrm{MHz}$ frequencies if direct-current (dc) biasing is used. Such attenuation poses a significant limitation to this method as the resonance frequencies are increased to the highfrequency range and beyond.

One way of handling this frequency-dependent transmission loss is to transform the impedance of the NEMS device down to $50 \mathrm{Ohm}$ by inserting appropriate circuitry before the transmission line. However, this may be difficult if the impedance mismatch ratio is greater than 100 and impractical if the same detection setup is to be used with many devices of different frequencies. Using high-frequency $50-\mathrm{Ohm}$ equipment without impedance matching, on the other hand, results in severe signal attenuation because of the high-ratio voltage divider formed by the high-impedance NEMS and the lowinput-impedance read-out circuitry. In this letter, we describe a way to circumvent these problems of impedance mismatch and extend the usability of low-frequency, high-inputimpedance measurement equipment.

Our technique utilizes ac biasing and the intrinsic properties of the piezoresistor to perform heterodyne downmixing of the signal to a much lower frequency, which can then be detected by standard circuitry without significant signal loss. Not only does this increase the detected signal, it also greatly reduces unwanted background from the cross-talk between the detector and actuator circuits. As proof of principle, we have applied this downmixing scheme to the detection of cantilever NEMS with fundamental mode frequencies of 5-25 MHz. We achieve thermal noise-limited detection of mechanical resonances in these devices at room temperature, demonstrating the ultimate sensitivity of downmixed piezoresistive signal detection in resonant high-frequency NEMS applications.

Figure 1(a) illustrates the difficulties in applying lowfrequency techniques naively to high-frequency piezoresistive NEMS. In most applications, the piezoresistor $R_{c}$ is

(a)

(b)
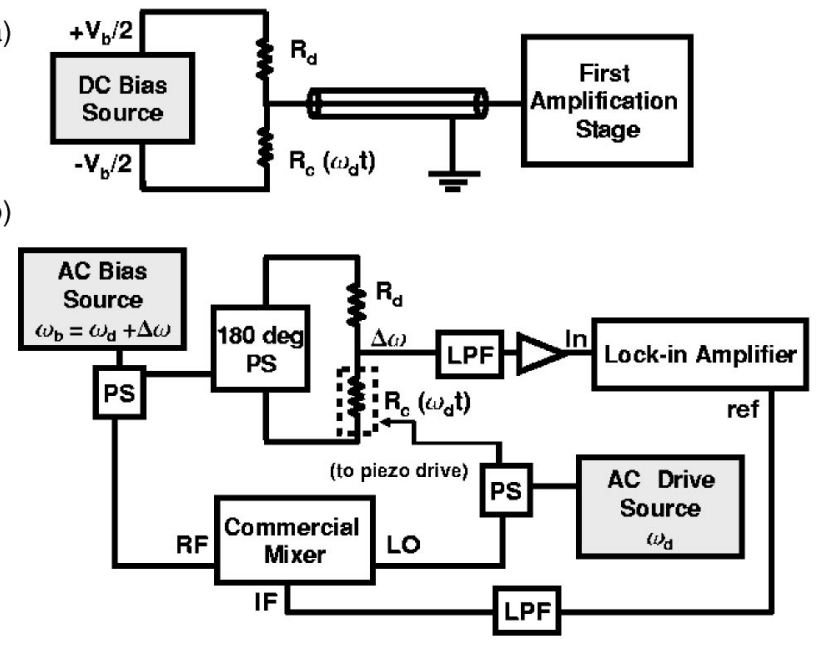

FIG. 1. Diagrams of direct-current bias (a) and downmixing (b) circuits. See text for details. 
placed in a bridge or half-bridge configuration with a fixed dummy resistor $R_{d}$; for simplicity we will assume $R_{c}=R_{d}$ $=R$ when the NEMS is at rest. The ends of the resistors are oppositely dc-biased at $+V_{b} / 2$ and $-V_{b} / 2$, so that the voltage at the bridge point is zero when the NEMS is not moving. When the NEMS is driven at a frequency $\omega_{d}, R_{c}$ gains a time-dependent component: $R_{c}=R+\Delta R \cos \left(\omega_{d} t+\phi\right)$; typically $\Delta R / R \leqslant 10^{-5} .{ }^{11}$ This resistance variation produces the standard resistance-bridge voltage $V_{\text {out }} \approx V_{b}(\Delta R / 4 R) \cos \left(\omega_{d} t\right.$ $+\phi)$ at the bridge point.

To measure $V_{\text {out }}$ the bridge output must be connected in some way to a circuit, e.g., the input of a high-inputimpedance preamplifier. At moderately high frequencies $(<30 \mathrm{MHz})$, this introduces capacitances $C_{\mathrm{par}}$ to ground in parallel with the cantilever and dummy, effectively forming a low-pass filter with a cut-off frequency of $\left(\pi R C_{\mathrm{par}}\right)^{-1}$. With typical amplifier-input and cable capacitances $C_{\mathrm{par}} \geqslant 10 \mathrm{pF}$, and $R \geqslant 10 \mathrm{k} \Omega$, the ac output is strongly attenuated at $\omega_{d} / 2 \pi>2 \mathrm{MHz}$. At very high frequencies $(>30 \mathrm{MHz})$, the measurements are further complicated by cable resonances.

Our circuit, depicted in Fig. 1(b), avoids these problems by using the piezoresistor to downmix the displacement signal to a lower frequency. This is achieved by applying an ac voltage $V_{b}(t)=V_{b 0} \cos \left(\omega_{b} t\right)$ across the resistors at a frequency $\omega_{b}$, offset from the drive frequency by an amount $\Delta \omega \equiv \omega_{b}-\omega_{d}$. Using a $180^{\circ}$ power splitter (PS), the voltage is applied oppositely to the ends of the resistors, to null the bias voltage at the bridge point. ${ }^{15}$ The bias produces an ac current $I(t)=V_{b 0} \cos \left(\omega_{b} t\right) /\left(R_{d}+R_{c}\right)$; to leading order in $\Delta R / R, V_{\text {out }}$ then becomes

$$
\begin{aligned}
V_{\text {out }}(t) & \approx \frac{V_{b 0} \cos \left(\omega_{b} t\right)}{4 R}\left(\Delta R \cos \left(\omega_{d} t+\phi\right)\right) \\
& =V_{b 0} \frac{\Delta R}{8 R}\left[\cos (\Delta \omega t-\phi)+\cos \left(\left(2 \omega_{d}+\Delta \omega\right) t+\phi\right)\right] .
\end{aligned}
$$

Thus the output signal at the bridge point contains two frequency components, at the sum and difference of the drive and bias frequencies. With $\Delta \omega$ sufficiently small $(<100 \mathrm{kHz})$, the downmixed frequency component is attenuated minimally by the parallel capacitances. The output is then sent through a low pass filter (LPF) to remove any residual carrier and the upper sideband, amplified, and finally fed into a lock-in amplifier for detection. The lock-in reference is generated by splitting off the bias and drive voltages with power splitters and sending the voltages into a commercial mixer, which generates a downmixed signal in parallel with the NEMS.

This electromechanical downmixing effect, while functionally similar to that of commercial diode-based mixers, is a fully linear property of the piezoresistor. Unlike regular mixers, where a minimum threshold signal is required to access the nonlinear response, the piezoresistor downconverts any signal to an amplitude half that of the signal that would be generated with a dc bias of the same magnitude. In contrast, any parasitic cross-talk signal from the actuation circuit would need to pass through an element with a nonlinear $I-V$ response in order to mix with the bias voltage down to the frequency $\Delta \omega$. We have found that the $I-V$ nonlinearity of our piezoresistors is quite small, so that even extremely weak displacement signals can be downmixed and extracted from the device with minimal attenuation and background. Downloaded 14 Dec 2005 to 131.215.225.171. Redistribution subject

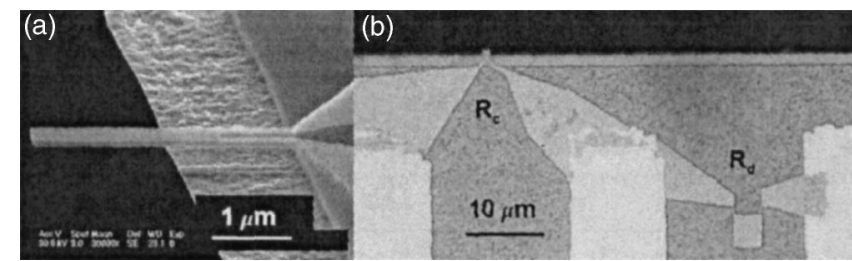

FIG. 2. (a) Scanning electron micrograph of a cantilever of dimensions $3.2 \mu \mathrm{m}$ long $\times 700 \mathrm{~nm}$ wide $\times 110 \mathrm{~nm}$ thick. (b) Optical micrograph of a cantilever and dummy resistor. Three gold leads at the bottom of the image connect the resistors to the rest of the circuit.

We have tested this downmixing scheme using highfrequency piezoresistive cantilevers as the NEMS device. The cantilevers are fabricated from silicon-on-insulator (SOI) wafers, where the top Si layer consists of $80 \mathrm{~nm} \mathrm{Si}$ plus a $30 \mathrm{~nm}$ layer of boron-doped $p$-Si to act as the piezoresistive strain sensor. The cantilevers were fabricated in a manner similar to that of Harley and Kenny, ${ }^{10}$ where a backside $\mathrm{KOH}$ etch suspends the top Si layer as a membrane, and a combination of electron beam lithography, liftoff, and fluorine/chlorine-based plasma etching steps forms the cantilever from the membrane. The particular details of our fabrication method are described elsewhere. ${ }^{16}$ A typical cantilever is shown in Fig. 2(a); lengths ranged from 2 to $3 \mu \mathrm{m}$ and widths were approximately $700 \mathrm{~nm}$. As shown in Fig. 2(b), the dummy resistor was fabricated on-chip using the same $p$-Si material that provides the cantilever strain sensor, in order to minimize parallel capacitances. The cantilever and dummy resistances varied from device to device, and were in the range of $50-150 \mathrm{k} \Omega$. The cantilever chip was mounted onto a piezoelectric ceramic actuator disk with a thickness of approximately $80 \mu \mathrm{m}$, corresponding to a thickness-mode resonance frequency of about $25 \mathrm{MHz}$. This assembly was in turn mounted onto a circuit board and placed into a vacuum chamber for measurements at room temperature.

Figure 3(a) displays a resonance curve for a 1.7- $\mu \mathrm{m}$ long cantilever using our downmixing method. Here $V_{b 0}=3 \mathrm{~V}$, $\Delta \omega / 2 \pi=100 \mathrm{kHz}$, and a peak-to-peak voltage of $1.9 \mathrm{~V}$ is applied to the piezoactuator. The resonance is approximately $f_{c}=24.4 \mathrm{MHz}$, close to but somewhat below the theoretically predicted fundamental-mode resonance frequency of $27 \mathrm{MHz}$. The square of the voltage signal is proportional to
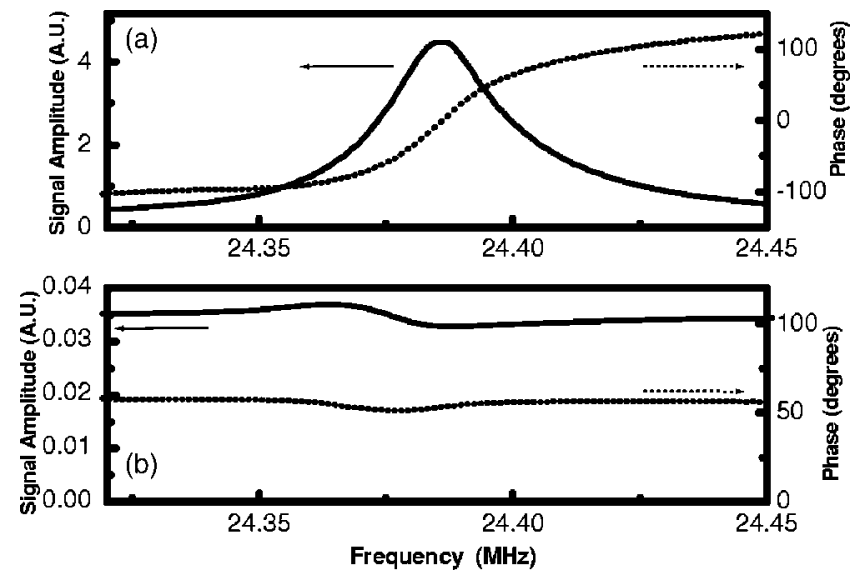

FIG. 3. (a) Signal amplitude (solid line) and phase (dotted line) of resonance of first vibration mode of a 1.7- $\mu$ m-long cantilever, as measured using downmixing with an offset frequency $\Delta \omega / 2 \pi=100 \mathrm{kHz}$. (b) Same measured directly with a network analyzer.

to AIP license or copyright, see http://apl.aip.org/apl/copyright.jsp 


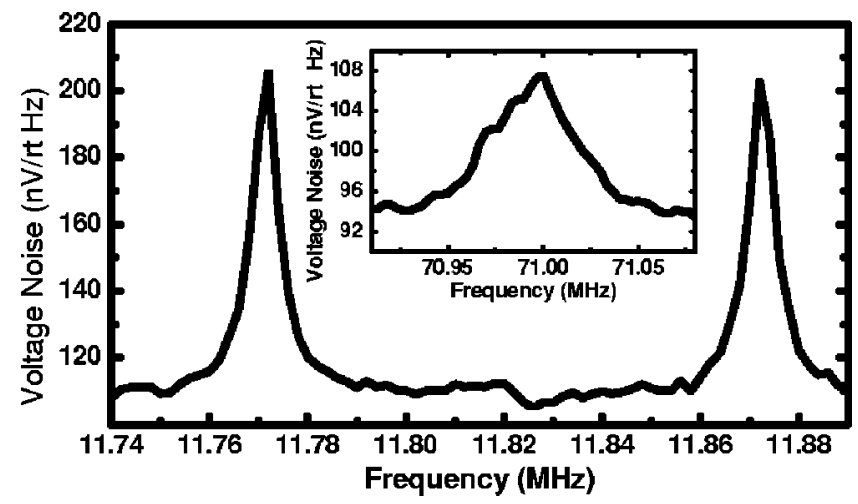

FIG. 4. Thermomechanical noise peaks from the fundamental vibration mode of a 2.6- $\mu \mathrm{m}$-long cantilever. Inset: One of the thermomechanical noise peaks from the second vibration mode of the same cantilever.

the energy in the cantilever; fitting this quantity to a Lorentzian yields a width of $18.2 \mathrm{kHz}$, implying a quality factor of $Q \approx 1300$. For comparison, Fig. 3(b) shows the same cantilever resonance measured in the standard dc half-bridge configuration, ${ }^{9}$ using a dc bias of $5 \mathrm{~V}$ across $R_{c}$ and $R_{d}$ and a 50-Ohm high-frequency network analyzer for drive (approximately $1 V_{p p}$ ) and detection. In both measurements no impedance matching had been performed, and the signal was normalized by the drive and bias levels. The resonant signal in the downmixing scheme is approximately 1000 times larger than in the direct measurement scheme. In addition, the relative magnitude of the background, caused by parasitic coupling of the drive signal to the detection circuit, is nearly three orders of magnitude smaller in the downmixing case. As a result, the downmixed resonance is essentially Lorentzian, while the network analyzer resonance is highly distorted.

This technique is sensitive enough to detect the thermomechanical fluctuations of the cantilever when it is not driven externally. This is done by applying only a bias voltage and, while sweeping the bias, detecting narrowband noise at the offset frequency $\Delta \omega$. Figure 4 shows the thermomechanical noise for a 2.6- $\mu \mathrm{m}$-long cantilever with fundamental mode frequency $f_{c}=11.9 \mathrm{MHz}$, using $\Delta \omega / 2 \pi$ $=50 \mathrm{kHz}$. The noise appears as two peaks spaced $100 \mathrm{kHz}$ apart because the noise in the cantilever at the resonance frequency is mixed to the $50 \mathrm{kHz}$ reference when the bias is either $50 \mathrm{kHz}$ above or below the cantilever resonance. The peak noise amplitude agrees with the theoretical estimate based on the previously measured piezoresistive gauge factor of similar cantilever devices. ${ }^{16}$ Note that the thermal conduction calculations predict the cantilever to be approximately $20 \mathrm{~K}$ hotter than the room-temperature environment due to Joule heating of the piezoresistor in this experiment. The off-resonance noise floor of $110 \mathrm{nV} / \sqrt{\mathrm{Hz}}$ is approximately twice that expected from a combination of thermomechanical noise and Johnson noise (the preamplifier input noise, at $0.6 \mathrm{nV} / \sqrt{\mathrm{Hz}}$, is negligible). The precise origin of this excess noise is unclear; however, we have found that we can reduce the noise floor to the expected Johnson noise level $(\sim 60 \mathrm{nV} / \sqrt{\mathrm{Hz}})$ by increasing the offset frequency and decreasing the bias voltage, while still maintaining the visibility of the thermomechanical noise peak on resonance. We note by comparison that our network analyzer measurement has input-referred noise amplitude of about $7 \mathrm{nV} / \sqrt{\mathrm{Hz}}$. The thermomechanical noise peak, attenuated by more than three orders of magnitude because of the low 50-Ohm input impedance of the network analyzer, would be nearly undetectable against this background.

The fact that the peak noise amplitude in Fig. 4 is about twice larger than the off-resonant noise floor allows us to conclude that the thermal excitation is the main source of noise in resonant experiments with this device. The corresponding force sensitivity can be calculated ${ }^{17}$ by using a value of the spring constant $k \approx 0.5 \mathrm{~N} / \mathrm{m}$ determined from the device dimensions and the elastic properties of $\mathrm{Si} ;{ }^{9}$ when Johnson noise is included, the overall resonant force sensitivity at $11.9 \mathrm{MHz}$ is $F_{N} \approx 350 \mathrm{aN} / \sqrt{\mathrm{Hz}}$ at room temperature, comparable to the lower-frequency cantilever devices studied previously. ${ }^{10}$

The thermomechanical noise peak remains visible in the downmixed scheme at even higher frequencies, as demonstrated by detection of the thermal noise peak from the second vibration mode of the 11.9-MHz cantilever (inset to Fig. 4 ), whose driven resonance we have detected at approximately $71 \mathrm{MHz}$. This demonstrates that the technique as presented here is viable for even smaller NEMS whose fundamental modes lie in the VHF range. In addition, while our measurements were limited to room temperature, we remark that both Johnson and thermomechanical voltage noise vary with temperature $T$ as $\sqrt{T}$, so that noise-limited detection of piezoresistive NEMS should scale well to lower temperatures.

The authors thank S. Stryker for assistance with construction of the experimental apparatus. We are grateful for support of this work from the NSF (ECS-0089061) and DARPA (DSO-MOSAIC N00014-02-1-0602 and MTO/ SPAWAR N66001-02-1-8914).

${ }^{1}$ M. L. Roukes, Phys. World 14, 25 (2001).

${ }^{2}$ A. N. Cleland and M. L. Roukes, Appl. Phys. Lett. 69, 2653 (1996).

${ }^{3}$ D. W. Carr, S. Evoy, L. Sekaric, H. G. Craighead, and J. M. Parpia, Appl. Phys. Lett. 75, 920 (1999).

${ }^{4}$ X. M. H. Huang, C. A. Zorman, M. Mehregany, and M. L. Roukes, Nature (London) 421, 496 (2003).

${ }^{5}$ A. N. Cleland and M. L. Roukes, Nature (London) 392, 160 (1998)

${ }^{6}$ K. L. Ekinci, X. M. H. Huang, and M. L. Roukes, Appl. Phys. Lett. 84, 4469 (2004).

${ }^{7}$ J. A. Sidles, J. L. Garbini, K. J. Bruland, D. Rugar, O. Zuger, S. Hoen, and C. S. Yannoni, Rev. Mod. Phys. 67, 249 (1995).

${ }^{8}$ M. D. Lahaye, O. Buu, B. Camarota, and K. C. Schwab, Science 304, 74 (2004).

${ }^{9}$ M. Tortonese, R. C. Barrett, and C. F. Quate, Appl. Phys. Lett. 62, 834 (1993).

${ }^{10}$ J. A. Harley and T. W. Kenny, Appl. Phys. Lett. 75, 289 (1999).

${ }^{11}$ H. J. Mamin, R. P. Ried, B. D. Terris, and D. Rugar, Proc. IEEE 87, 1014 (1999).

${ }^{12}$ R. L. Gunter, W. G. Delinger, K. Manygoats, A. Kooser, and T. L. Porter, Sens. Actuators, A 107, 219 (2003).

${ }^{13}$ Y. Kanda, IEEE Trans. Electron Devices 29, 64 (1982).

${ }^{14}$ K. Hjort, J. Soderkvist, and J. Schweitz, J. Micromech. Microeng. 4, 1 (1994).

${ }^{15}$ K. L. Ekinci, Y. T. Yang, X. M. H. Huang, and M. L. Roukes, Appl. Phys. Lett. 81, 2253 (2002).

${ }^{16} \mathrm{~J}$. Arlett and M. L. Roukes (unpublished).

${ }^{17}$ A. N. Cleland and M. L. Roukes, J. Appl. Phys. 92, 2758 (2002). 